\title{
The Henize sample of S stars
}

\section{New symbiotic stars ${ }^{\star}$}

\begin{abstract}
S. Van Eck ${ }^{\star \star}$ and A. Jorissen ${ }^{\star \star \star}$
Institut d'Astronomie et d'Astrophysique, Université Libre de Bruxelles, CP 226, Boulevard du Triomphe, 1050 Bruxelles, Belgium

Received 24 June 2002 / Accepted 20 September 2002

Abstract. The properties of the few symbiotic stars detected among the 66 binary S stars from the Henize sample are discussed. Two stars (Hen 18 and Hen 121) exhibit both a strong blue-violet continuum and strong $\mathrm{H}_{\alpha}$ emission ( $F W H M$ of $70 \mathrm{~km} \mathrm{~s}^{-1}$ ), whereas Hen 134 and 137 exhibit weak $\mathrm{H}_{\alpha}$ emission. The $\mathrm{H}_{\alpha}$ profiles are typical of non-dusty symbiotic stars belonging to class S-3 as defined by Van Winckel et al. (1993, A\&AS 102, 401). In that class as in the Henize symbiotic S stars, He I, [N II] or [S II] emission lines are absent, suggesting that the nebular density is high but the excitation rather low. The radial velocity of the centre of the $\mathrm{H}_{\alpha}$ emission is identical to that of the companion star (at least for Hen 121 where this can be checked from the available orbital elements), thus suggesting that the $\mathrm{H}_{\alpha}$ emission originates in gas moving with the companion star. For Hen 121, this is further confirmed by the disappearance of the ultraviolet Balmer continuum when the companion is eclipsed by the S star. Hen 121 is thus the second eclipsing binary star discovered among extrinsic S stars (the first one is HD 35155). A comparison of the available data on orbital periods and $\mathrm{H}_{\alpha}$ emission leads to the conclusion that $\mathrm{H}_{\alpha}$ emission in $\mathrm{S}$ stars seems to be restricted to binary systems with periods in the range 600-1000 d, in agreement with the situation prevailing for red symbiotic stars (excluding symbiotic novae). Symbiotic S stars are found among the most evolved extrinsic S stars.
\end{abstract}

Key words. stars: binaries: symbiotic - stars: AGB and post-AGB - stars: late-type - accretion, accretion disks - line: profiles stars: binaries: eclipsing

\section{Introduction}

The defining spectral properties of symbiotic and S stars are very different, and do not a priori hint at a possible relationship between them. Symbiotic stars exhibit at the same time spectral features typical of cool stars (like molecular bands), of hot stars (like an ultraviolet continuum) and of nebulae (like forbidden emission lines) (see Kenyon 1986). Stars of type S are red giants with $\mathrm{ZrO}$ bands in their spectra. However, technetiumpoor $^{1}$ (or "extrinsic") S stars (see Jorissen et al. 1998; Van Eck \& Jorissen 2000b, for recent reviews) and symbiotic stars share similarities in that they are two classes of binary stars involving a red giant and a white dwarf companion in relatively wide systems with orbital periods larger than about $200 \mathrm{~d}$. This similarity naturally raises the question of the relationship between these two families, or in other words, why do those binary

Send offprint requests to: S. Van Eck, e-mail: svaneck@astro.ulb.ac.be

* Based on observations carried out at the European Southern Observatory (ESO, La Silla, Chile; program 60.E-0805) and at the Swiss $70 \mathrm{~cm}$ telescope (La Silla, Chile).

$\star \star$ Post-doctoral Researcher, F.N.R.S., Belgium.

$\star \star \star$ Research Associate, F.N.R.S., Belgium.

1 Tc-rich (also called "intrinsic") $\mathrm{S}$ stars are genuine asymptotic giant branch stars that, unlike Tc-poor $\mathrm{S}$ stars, are not necessarily binaries, and are therefore not relevant to the present study. systems seem to appear in two different flavours? This question is actually twofold: (i) Do symbiotic systems exhibit the same kind of abundance peculiarities as S stars, namely enhanced carbon and elements heavier than Fe produced by the s-process of nucleosynthesis? (ii) Do Tc-poor S stars exhibit symbiotic activity?

To answer the first question, it is necessary to distinguish yellow symbiotics (i.e., involving a $\mathrm{G}$ or $\mathrm{K}$ giant) from red symbiotics (i.e., involving an $\mathrm{M}$ giant). All detailed abundance studies performed so far for yellow symbiotics have revealed heavy-element overabundances resembling those observed in S stars (Schmid 1994; Smith et al. 1996, 1997; Pereira \& Porto de Mello 1997; Pereira et al. 1998; Smith et al. 2001; Pereira et al. 2003). On the contrary, not a single red symbiotic star from the catalogue of Mürset \& Schmid (1999) seems to exhibit the $\mathrm{ZrO}$ distinctive spectral features of S-type stars. This puzzling situation is reviewed by Jorissen (2003).

The present paper deals with the question of the possible symbiotic activity among $\mathrm{S}$ stars. Several previous studies have indeed identified S stars with symbiotic activity (as will be reviewed in Sect. 4.3), but those previously known cases were isolated cases, not resulting from a systematic search. Hence they do not provide much insight into the frequency of this phenomenon among S stars. In this paper, we report the result of a search for $\mathrm{H}_{\alpha}$ emission among a sample of 29 supposedly 
binary S stars identified by Van Eck \& Jorissen (2000a,b) (hereafter denoted by Papers II and III, respectively) in the Henize sample of $205 \mathrm{~S}$ stars.

\section{Binary $S$ stars among the Henize sample}

The Henize sample of S stars has been assembled by K. Henize from the objective-prism plates of the Michigan-Mount Wilson survey of the southern sky for $\mathrm{H}_{\alpha}$ emission objects. Besides this primary aim, Henize readily realized that the plates could also be used to identify $\mathrm{S}$ stars (not necessarily with $\mathrm{H}_{\alpha}$ in emission). His list of S stars, that he never published however, can be traced through Stephenson's General Catalogue of Galactic S Stars (Stephenson 1984), where they appear as Henun $n n n^{2}$ (with $n n n \leq 205$ ). The major advantage of the Henize sample is that it is not restricted to the galactic plane, since it comprises the $205 \mathrm{~S}$ stars south of declination $-25^{\circ}$ that are brighter than $R=10.5$ (the completeness of the sample has been discussed in Paper III). Therefore, the sample is not biased against extrinsic, Tc-poor, binary S stars, which are not as concentrated along the galactic plane as are intrinsic S stars (Paper III). The identification of the extrinsic S stars in the Henize sample has been performed in Papers II and III using multivariate classification analysis relying on the radial-velocity dispersion, the $U-B$, $B-V, V-K$, and IRAS $K-[12]$ color indices, as well as on the $\mathrm{ZrO}$ and $\mathrm{TiO}$ band strengths, on the presence/absence of Tc and on the photometric variability. This classification procedure yielded 66 extrinsic S stars out of 199 S stars with available data (see Table 6 of Paper II). The binary nature of those stars is strongly suggested by the large dispersion of their radial velocities (Fig. 10 of Paper III), while orbits are available for 5 of those stars (Table 1 of Paper II).

Among these 66 binary stars, two (namely Hen 18 and Hen 121) stand out because of their very blue colors (see Sect. 3.2 and Fig. 3 of Paper III). Figure 1 presents the evolution of the color indices of these two stars in the dereddened ${ }^{3}$ $(U-B, B-V)$ diagram. This evolution is very similar to the one of classical symbiotic stars (see e.g., Arkhipova \& Noskova 1985). Table 1 lists the individual photometric measurements in the Geneva system, which are too sparse to derive a meaningful lightcurve. For Hen 121, our photometric data nevertheless contain the clear signature of an eclipse of the ultraviolet Balmer continuum by the $\mathrm{S}$ star. This is clearly visible in Table 1 (numbers in bold face) and in the inset of Fig. 1, which reveal that the $U-B$ color of Hen 121 becomes much redder at $\mathrm{JD}=2449053.76,2449068.75$ and 2449074.73 , and is then typical of non-symbiotic, extrinsic S stars. The orbital elements listed in Paper II indicate that these measurements were taken at phases $0.70,0.72$ and $0.73( \pm 0.01)$, while the spectroscopic ephemeris predicts the eclipse of the companion by the $\mathrm{S}$ star to be central at phase 0.75 . This provides a clear indication that the Balmer continuum observed in the Hen 121 system is tied to the companion. Hen 121 is thus the second eclipsing binary star

\footnotetext{
2 In SIMBAD, they are referred to as Hen 4-nnn.

3 The dereddening procedure applied to the data of Table 1 to obtain Fig. 1 has been described in Sect. 3.3 of Paper II.
}

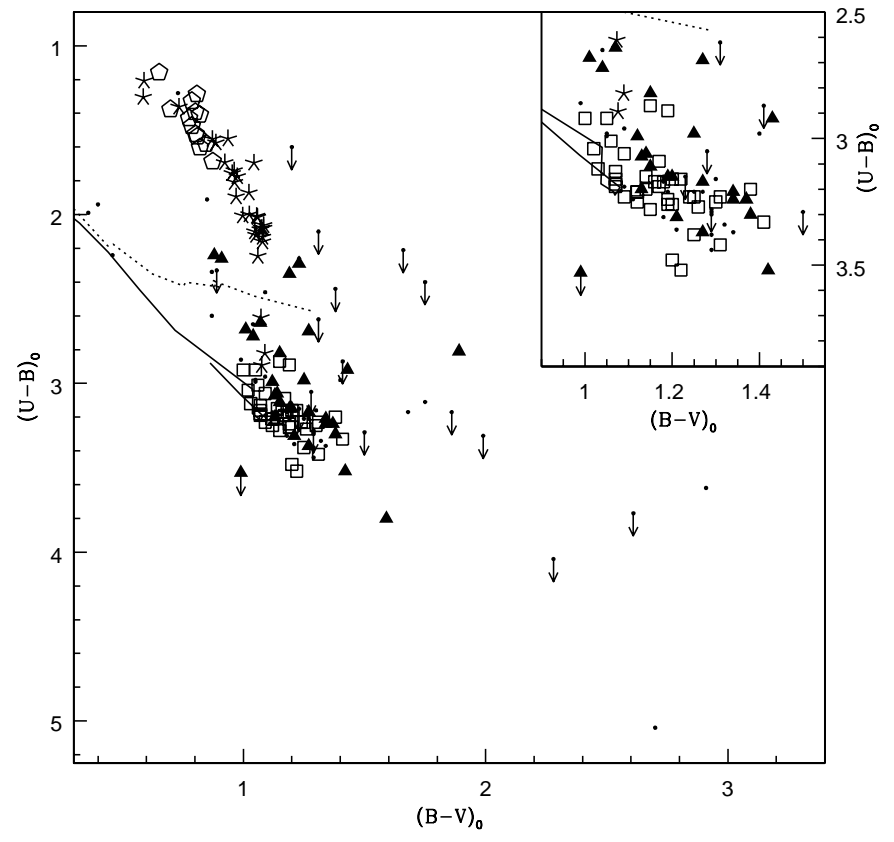

Fig. 1. Dereddened $(U-B, B-V)$ diagram, in the Geneva photometric system, for non-symbiotic $S$ stars (average of individual measurements) and symbiotic $\mathrm{S}$ stars (individual measurements). Tc-rich, intrinsic $\mathrm{S}$ stars and Tc-poor, extrinsic (binary) $\mathrm{S}$ stars are represented by filled triangles and open squares, respectively, whereas dots denote stars with unknown Tc content. Stars with only upper limits on their $U$ flux are flagged with an arrow. The individual measurements of the symbiotic S stars Hen 18 and 121 are represented by open hexagons and star symbols, respectively. Note how the color sequence of Hen 121 extends down into the region occupied by the extrinsic S stars. The three measurements of Hen 121 located in the inset were taken during the eclipse of the companion by the $\mathrm{S}$ star. The solid and dotted lines are the normal giant and dwarf sequences, respectively, from Grenon (1978).

discovered among extrinsic S stars (the first one is HD 35155; Jorissen et al. 1992).

The time of first contact (which occurred between JD = 2449044.83 and JD $=2449053.76$ according to Table 1 and Fig. 1, corresponding to phases 0.69 and 0.70 , respectively) makes it possible to estimate the $\mathrm{S}$-star radius. Adopting $\phi=0.695$ for the phase of first contact, $\sin i=1, M_{\mathrm{S}}=1 M_{\odot}$ and $M_{\mathrm{WD}}=0.6 M_{\odot}$ for the S-star and WD masses, respectively (as suggested by Fig. 3 below and consistent with the mass function $0.092 M_{\odot}$ for the system; see Paper I), the radius of the $\mathrm{S}$ star amounts to $R=153 R_{\odot}$, and its Roche lobe to $180 R_{\odot}$. Hence Hen 121 is close to filling its Roche lobe.

This radius, combined with an effective temperature of $3400 \pm 100 \mathrm{~K}$, as derived from the $\left[T_{\text {eff }},(V-K)_{0}\right]$ calibration of Plez et al. (2003) with $(V-K)_{0}=6.1$, yields $M_{\text {bol }}=-3.9$. This combination of bolometric magnitude and effective temperature locates Hen 121 among the most evolved extrinsic S stars, according to Fig. 2 of Van Eck et al. (1998). The mass loss rate of Hen 121 may concomittantly be expected to be large, a fundamental requirement to trigger symbiotic activity as discussed in Sect. 4.3.

The high-resolution $\mathrm{H}_{\alpha}$ spectroscopic survey of extrinsic $S$ stars presented in this paper confirms that the two stars 
Table 1. Geneva photometry of Hen 18 and Hen 121. IPV and IPC are quality indices for the $V M$ magnitude and for the colors, respectively, in a scale from 0 (bad) to 4 (excellent). Data in bold case correspond to the eclipse of the Balmer ultraviolet continuum of Hen 121.

\begin{tabular}{|c|c|c|c|c|c|c|c|c|c|c|}
\hline $\begin{array}{c}\text { JD } \\
(-2440000)\end{array}$ & $\overline{~ I P V}$ & $\overline{V M}$ & IPC & $\overline{U-B}$ & $\overline{V-B}$ & $\overline{P B 1-B}$ & $\overline{P B 2-B}$ & $\bar{V} V 1-B$ & $\overline{G-B}$ & "airmass \\
\hline \multicolumn{11}{|l|}{ Hen 18} \\
\hline 8686.573 & 3 & 10.488 & 3 & 1.288 & -0.852 & 1.196 & 1.208 & -0.041 & 0.085 & 1.02 \\
\hline 8718.558 & 3 & 10.590 & 3 & 1.559 & -0.976 & 1.297 & 1.177 & -0.167 & -0.045 & 1.21 \\
\hline 9428.543 & 2 & 10.508 & 3 & 1.668 & -1.010 & 1.331 & 1.141 & -0.195 & -0.078 & 1.02 \\
\hline 10148.577 & 3 & 10.605 & 3 & 1.814 & -1.073 & 1.413 & 1.158 & -0.249 & -0.143 & 1.03 \\
\hline 10166.532 & 3 & 10.603 & 3 & 1.653 & -1.003 & 1.345 & 1.186 & -0.189 & -0.071 & 1.03 \\
\hline 10170.535 & 2 & 10.571 & 2 & 1.710 & -1.046 & 1.373 & 1.170 & -0.232 & -0.108 & 1.06 \\
\hline 10181.520 & 3 & 10.602 & 4 & 1.604 & -0.987 & 1.291 & 1.153 & -0.176 & -0.045 & 1.10 \\
\hline 10189.507 & 2 & 10.603 & 3 & 1.538 & -1.020 & 1.291 & 1.136 & -0.211 & -0.082 & 1.12 \\
\hline 10506.607 & 2 & 10.743 & 2 & 1.505 & -0.899 & 1.278 & 1.187 & -0.082 & 0.066 & 1.04 \\
\hline 10727.839 & 4 & 10.697 & 4 & 1.731 & -1.021 & 1.320 & 1.168 & -0.213 & -0.096 & 1.20 \\
\hline 10741.800 & 4 & 10.625 & 4 & 1.457 & -0.987 & 1.299 & 1.168 & -0.171 & -0.050 & 1.21 \\
\hline 10788.770 & 2 & 10.563 & 3 & 1.523 & -1.002 & 1.342 & 1.144 & -0.194 & -0.070 & 1.00 \\
\hline 10798.752 & 2 & 10.524 & 3 & 1.417 & -1.008 & 1.283 & 1.118 & -0.183 & -0.069 & 1.00 \\
\hline \multicolumn{11}{|l|}{ Hen 121} \\
\hline 8685.809 & 3 & 10.400 & 3 & 1.809 & -1.034 & 1.226 & 1.195 & -0.222 & -0.111 & 1.03 \\
\hline 8715.665 & 2 & 10.551 & 2 & 1.602 & -1.017 & 1.204 & 1.262 & -0.194 & -0.081 & 1.01 \\
\hline 9044.830 & 2 & 10.626 & 2 & 1.627 & -0.967 & 1.199 & 1.243 & -0.156 & 0.019 & 1.03 \\
\hline 9053.761 & 3 & 10.384 & 3 & 2.663 & -1.153 & 1.249 & 1.158 & -0.334 & -0.178 & 1.00 \\
\hline 9068.748 & 3 & 10.468 & 3 & 2.873 & -1.169 & 1.361 & 1.175 & -0.357 & -0.209 & 1.01 \\
\hline 9074.728 & 2 & 10.509 & 2 & 2.945 & -1.156 & 1.344 & 1.185 & -0.344 & -0.183 & 1.01 \\
\hline 10145.706 & 2 & 10.454 & 1 & 2.059 & -1.077 & 1.255 & 1.187 & -0.274 & -0.134 & 1.06 \\
\hline 10150.830 & 3 & 10.443 & 2 & 1.861 & -1.044 & 1.280 & 1.212 & -0.234 & -0.108 & 1.09 \\
\hline 10154.780 & 1 & 10.437 & 3 & 1.826 & -1.055 & 1.254 & 1.207 & -0.243 & -0.106 & 1.02 \\
\hline 10163.653 & 2 & 10.551 & 1 & 1.792 & -1.042 & 1.246 & 1.235 & -0.229 & -0.094 & 1.08 \\
\hline 10166.679 & 3 & 10.563 & 3 & 1.744 & -1.003 & 1.219 & 1.205 & -0.187 & -0.042 & 1.02 \\
\hline 10170.761 & 2 & 10.576 & 2 & 1.606 & -0.952 & 1.184 & 1.248 & -0.126 & 0.006 & 1.05 \\
\hline 10179.664 & 3 & 10.604 & 3 & 1.946 & -1.050 & 1.218 & 1.242 & -0.223 & -0.085 & 1.00 \\
\hline 10187.636 & 3 & 10.442 & 2 & 2.173 & -1.132 & 1.269 & 1.183 & -0.334 & -0.195 & 1.01 \\
\hline 10212.517 & 3 & 10.526 & 3 & 2.213 & -1.159 & 1.289 & 1.186 & -0.344 & -0.213 & 1.08 \\
\hline 10225.598 & 2 & 10.672 & 3 & 1.923 & -1.103 & 1.227 & 1.237 & -0.261 & -0.171 & 1.04 \\
\hline 10255.522 & 3 & 10.429 & 4 & 2.117 & -1.164 & 1.307 & 1.154 & -0.359 & -0.236 & 1.05 \\
\hline 10257.520 & 3 & 10.414 & 3 & 2.133 & -1.163 & 1.275 & 1.162 & -0.340 & -0.220 & 1.06 \\
\hline 10259.500 & 3 & 10.420 & 3 & 2.133 & -1.154 & 1.275 & 1.155 & -0.344 & -0.219 & 1.03 \\
\hline 10262.531 & 3 & 10.446 & 4 & 2.299 & -1.140 & 1.365 & 1.186 & -0.315 & -0.202 & 1.12 \\
\hline 10268.517 & 2 & 10.391 & 2 & 2.062 & -1.135 & 1.303 & 1.152 & -0.329 & -0.180 & 1.13 \\
\hline 10272.492 & 3 & 10.414 & 3 & 2.052 & -1.104 & 1.303 & 1.185 & -0.298 & -0.142 & 1.09 \\
\hline 10273.530 & 3 & 10.418 & 3 & 2.153 & -1.127 & 1.270 & 1.193 & -0.321 & -0.163 & 1.24 \\
\hline 10274.521 & 4 & 10.428 & 4 & 2.141 & -1.150 & 1.293 & 1.143 & -0.333 & -0.195 & 1.20 \\
\hline 10285.492 & 3 & 10.477 & 3 & 2.069 & -1.138 & 1.252 & 1.198 & -0.331 & -0.192 & 1.21 \\
\hline 10288.498 & 3 & 10.471 & 2 & 2.186 & -1.159 & 1.295 & 1.187 & -0.342 & -0.200 & 1.29 \\
\hline 10291.518 & 3 & 10.482 & 3 & 1.746 & -1.123 & 1.271 & 1.211 & -0.298 & -0.184 & 1.49 \\
\hline 10480.809 & 1 & 10.239 & 1 & 1.415 & -0.814 & 1.197 & 1.257 & -0.018 & 0.152 & 1.03 \\
\hline 10514.882 & 3 & 10.283 & 3 & 1.260 & -0.670 & 1.121 & 1.279 & 0.128 & 0.298 & 1.27 \\
\hline 10518.799 & 4 & 10.230 & 4 & 1.357 & -0.667 & 1.104 & 1.288 & 0.133 & 0.306 & 1.04 \\
\hline
\end{tabular}

standing out in the $(U-B, B-V)$ diagram of Fig. 1, namely Hen 18 and Hen 121, are the only two stars exhibiting clear symbiotic activity (two marginal cases - Hen 134 and Hen 137 - will be discussed in Sect. 4). In fact, when the multivariate classification analysis described in Paper III is allowed to go beyond the simple extrinsic/intrinsic dichotomy, the extrinsic sample is further subdivided in three subgroups, one of these (cluster \# 3 in Table 2 of Paper III) comprising the two symbiotic S stars with exceptionally blue and variable colors (see also Fig. 9 below).

\section{Observations}

High-resolution spectra (with $R=\lambda / \Delta \lambda=80000$ ) were obtained between February 21 and 27, 1998, with the $1.4 \mathrm{~m}$ CAT telescope of the European Southern Observatory 


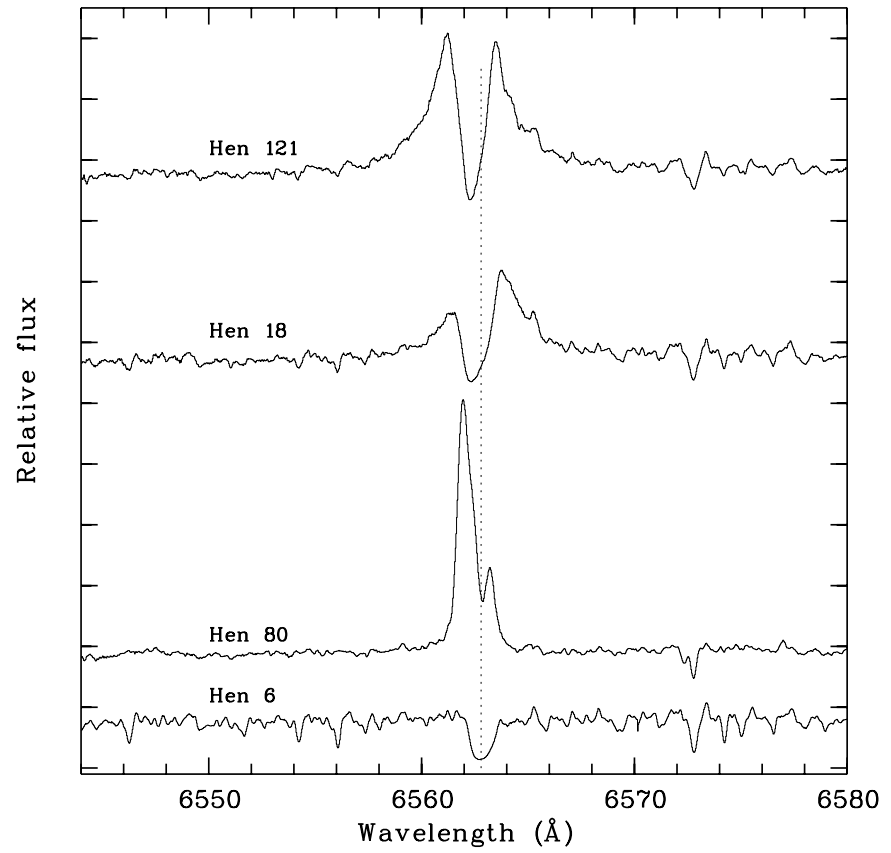

Fig. 2. The $\mathrm{H}_{\alpha}$ profiles of the two extrinsic $\mathrm{S}$ stars Hen 18 and 121, taken on February 21, 1998, compared to typical $\mathrm{H}_{\alpha}$ profiles for the intrinsic Mira S star Hen 80 and for the extrinsic S star Hen 6. The wavelength scale has been corrected from the radial-velocity of the stars, with the dashed line corresponding to the $\mathrm{H}_{\alpha}$ laboratory wavelength.

(ESO, La Silla, Chile), equipped with the Coudé Echelle Spectrograph (CES) and the $f / 4.7$ long camera. The CCD detector (ESO \# 38) is a Loral/Lesser back-illuminated, UV flooded thin chip with $2688 \times 512$ pixels of 15 by $15 \mu \mathrm{m}$. Spectra covering about $6 \mathrm{~nm}$ around the $\mathrm{H}_{\alpha}$ line $(\lambda 657.3 \mathrm{~nm})$ have been obtained for 29 extrinsic $\mathrm{S}$ stars. For the two symbiotic candidates (Hen 18 and 121), spectra were also obtained at the central wavelength $\lambda 669.7 \mathrm{~nm}$, covering [N II] $\lambda 654.8$ and $\lambda 658.3$, He I $\lambda 667.8$, and [S II] $\lambda 671.6$ and $\lambda 673.1$. Spectra with these two settings were also obtained for the S stars HD 35155 and HD 49368 that were previously reported to exhibit some symbiotic activity (see Sect. 4.3). At least two radial-velocity standard stars were observed each night to tie our wavelength scale to the IAU one.

\section{Analysis}

\section{1. $H_{\alpha}$ profiles}

Strong and broad $\mathrm{H}_{\alpha}$ emission lines were only observed for the two extrinsic $\mathrm{S}$ stars already suspected to be symbiotics from their $U B V$ color indices (Sect. 2 and Fig. 1). The corresponding profiles, compared to those of a typical Mira, intrinsic S star (Hen 80) and of a typical extrinsic S star (Hen 6), are shown in Fig. 2. The Hen 18 and Hen 121 profiles are indeed very different from those of Mira stars [see also Woodsworth (1995) for a discussion of $\mathrm{H}_{\alpha}$ emission profiles of Mira $\mathrm{S}$ stars], both in terms of the relative strengths of the red and blue emission peaks, and in terms of the base width. The Hen 18 and 121 profiles consist of a broad emission (half width at the base

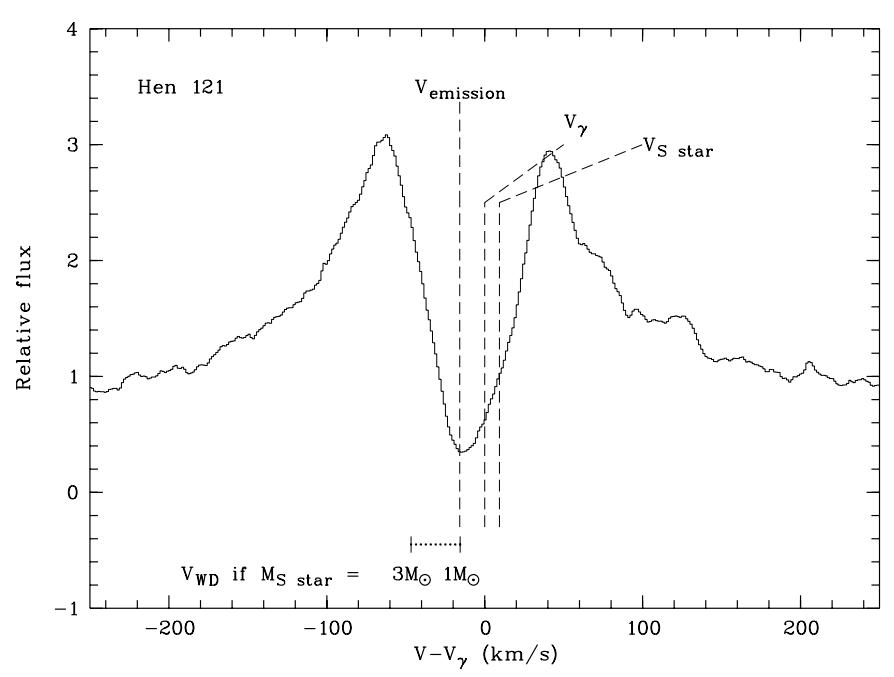

Fig. 3. The velocities of the center of mass of the system (denoted $V_{\gamma}$ ), of the $\mathrm{S}$ star (denoted $V_{\text {Star }}$ ) and of the companion star (as indicated by the horizontal segment corresponding to different values for $M_{\mathrm{S}}$, assuming $M_{\mathrm{WD}}=0.6 M_{\odot}$ ) have been located on the $\mathrm{H}_{\alpha}$ profile of Hen 121. The central wavelength of the emission (dashed line labeled $V_{\text {emission, }}$, as derived from a gaussian fit to the emission profile) is seen to coincide with the velocity of the companion, provided that $M_{\mathrm{S}}=1 M_{\odot}$ and $M_{\mathrm{WD}}=0.6 M_{\odot}$. The components' velocities have been computed from the orbital elements provided in Paper II, given the orbital phase of 0.08 , i.e., close to quadrature.

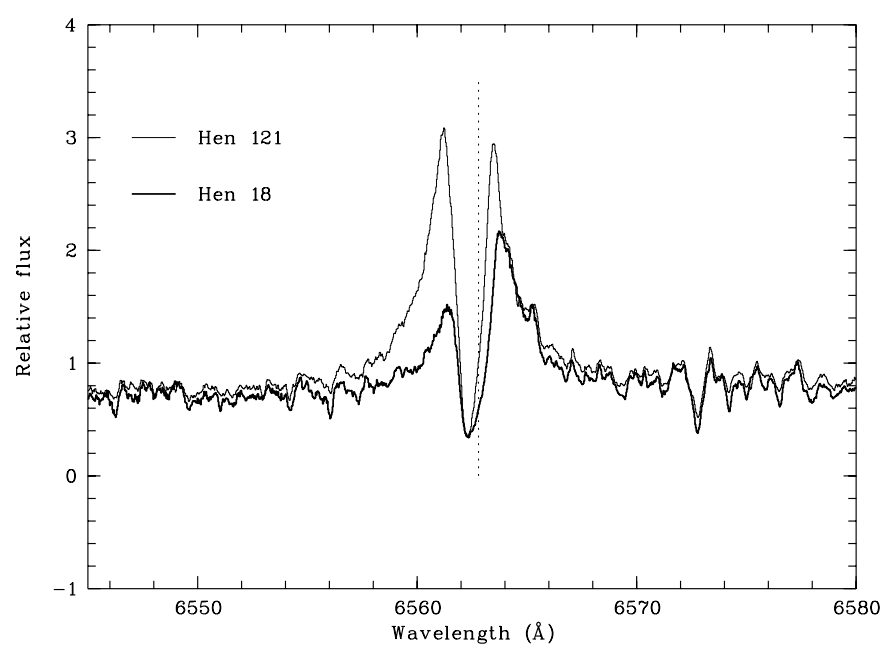

Fig. 4. Comparison of the $\mathrm{H}_{\alpha}$ profiles of Hen 18 and Hen 121

of about $250 \mathrm{~km} \mathrm{~s}^{-1}$, and $F W H M$ of $70 \mathrm{~km} \mathrm{~s}^{-1}$ ) with a central absorption core exactly centered on the emission (Fig. 3). Those $\mathrm{H}_{\alpha}$ profiles are similar to the $\mathrm{S}-3$ types identified by Van Winckel et al. (1993) in symbiotic stars. No He I, [N II] or [S II] emission lines are recorded in Hen 18 and 121, as expected for symbiotic stars with $\mathrm{H}_{\alpha}$ profiles of the S-3 kind (Table 6 of Van Winckel et al. 1993). The absence of these lines suggests that the associated nebula has a high density and a rather low excitation state. The S-3 class contains mainly recurrent and symbiotic novae.

Figure 4 compares the $\mathrm{H}_{\alpha}$ emission profiles of Hen 18 and Hen 121, and reveals striking similarities. The slight difference between these two profiles is reminiscent of the variations 
observed with orbital phase in symbiotic systems (for one particular example, see Fig. 4 of Mürset et al. 2000). Thus, similar formation mechanisms for $\mathrm{H}_{\alpha}$ must be operating in classical symbiotic stars and in symbiotic $\mathrm{S}$ stars. This mechanism is still debated. Two different processes have been proposed: (i) the whole $\mathrm{H}_{\alpha}$ profile is formed in the accretion disc forming around the accreting companion (see e.g., Robinson et al. 1994), (ii) the central dip of the $\mathrm{H}_{\alpha}$ profile results from absorption by the red-giant wind (Schwank et al. 1997; Mürset et al. 2000) while the broad $\mathrm{H}_{\alpha}$ wings are caused by Raman scattering of $\mathrm{Ly}_{\beta}$ photons from the hot component (Lee 2000).

The data available for Hen 18 and Hen 121 are too scarce to distinguish between these two formation processes, especially since the variation of the profile with orbital phase is not known. Let us just remark that, in Hen 121, the broad emission is almost exactly centered on the velocity of the companion star (see Fig. 3), provided that $M_{\mathrm{S}}=1 M_{\odot}$ and $M_{\mathrm{WD}}=0.6 M_{\odot}$. This pair is consistent with the mass function of the system $\left(0.092 \pm 0.013 M_{\odot}\right.$; see Table 1 of Paper II $)$, which predicts $M_{\mathrm{S}}=0.93 \pm 0.1 M_{\odot}$ for an eclipsing system with $\sin i \sim 1$ and $M_{\mathrm{WD}}=0.6 M_{\odot}$. Moreover, despite its blueshifted appearance, the absorption core is exactly centered on the emission (the false impression that it is blueshifted is actually caused by the presence of $\mathrm{H}_{\alpha}$ and $\mathrm{TiO}$ absorptions formed in the atmosphere of the $\mathrm{S}$ star and which mutilate the red wing of the $\mathrm{H}_{\alpha}$ emission). These properties of the $\mathrm{H}_{\alpha}$ profile tend to support the hypothesis that the $\mathrm{H}_{\alpha}$ emission originates from gas close to the companion. However, the similarity between the velocity of the emission and absorption components in Hen 18 and Hen 121, as revealed by Fig. 4, is difficult to understand in that framework, since it is very unlikely that the orbital phases at the time of observation - and hence the velocity of the companion star were identical in these two systems (this can unfortunately not be checked as orbital elements are not available for Hen 18).

\subsection{More symbiotic stars among Henize $S$ stars}

In an attempt to find weaker cases of symbiotic activity among binary $\mathrm{S}$ stars from the Henize sample, their $\mathrm{H}_{\alpha}$ profiles have been scrutinized in two different ways: (i) by deriving the residual profile after subtraction of the $\mathrm{H}_{\alpha}$ absorption normally present in $\mathrm{S}$ stars, and (ii) by examining the line bisector.

Figure 5 presents a few representative examples of the residual $\mathrm{H}_{\alpha}$ profile obtained after subtracting the spectrum of Hen 6, chosen as a template because its bisector appears to be very clean (see Fig. 6 below). Two new cases of S stars with symbiotic-like $\mathrm{H}_{\alpha}$ profiles emerge from this figure: Hen 134 and 137. The case of Hen 129 and Hen 138, also shown in Fig. 5, is less clear: the weak emission remaining after subtraction of the Hen 6 template (Fig. 5) is more likely due to a template mismatch than to a real $\mathrm{H}_{\alpha}$ emission.

The P-Cygni shape of the residual $\mathrm{H}_{\alpha}$ profile appearing on Fig. 5 for Hen 134 and 137 may also be inferred from their line bisectors displayed on Fig. 6, which are quite different from those of non-symbiotic stars. Almost vertical, straight bisectors are found for the G giant HD 36079 and the early M giant $\gamma \mathrm{Cru}$, as well as for the extrinsic S star Hen 6 (leftmost column

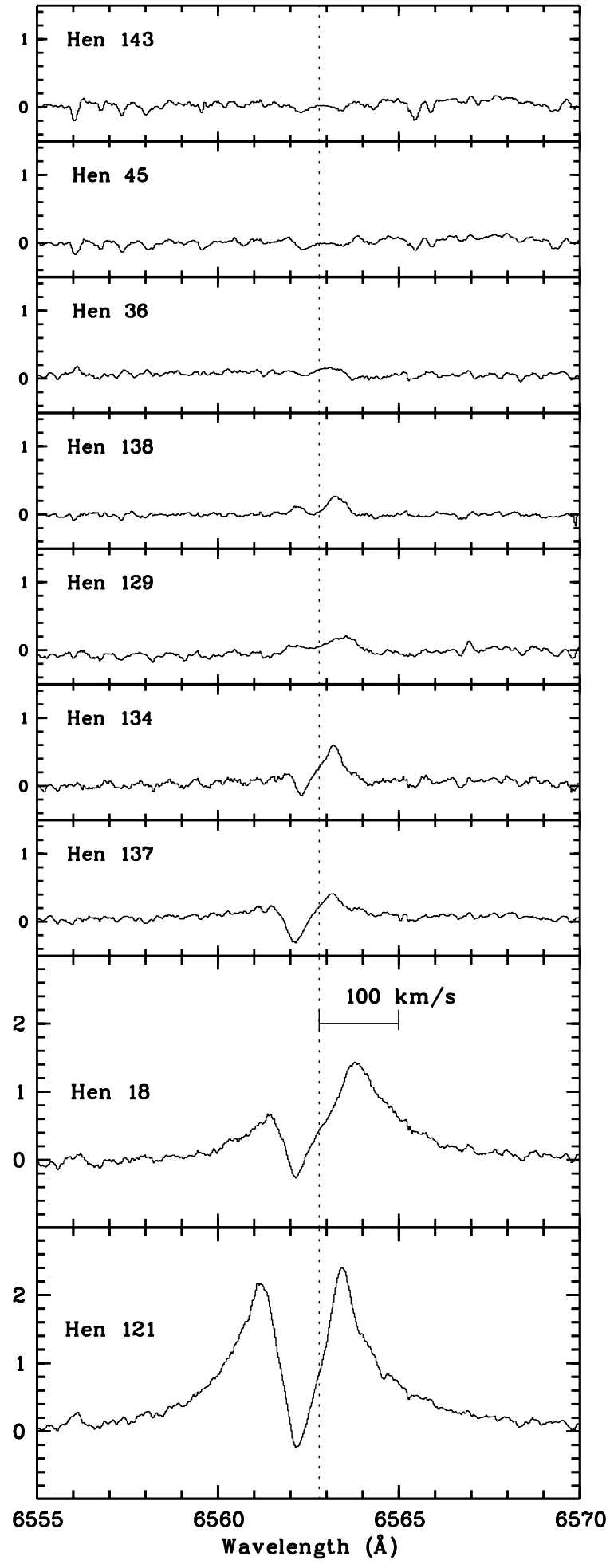

Fig. 5. Residual $\mathrm{H}_{\alpha}$ profiles obtained after subtracting the Hen 6 profile, for a few representative cases (from top to bottom, ordered according to the increasing strength of their $\mathrm{H}_{\alpha}$ emission): (i) extrinsic star with no residual $\mathrm{H}_{\alpha}$ emission/absorption (Hen 143); (ii) intrinsic stars where residual $\mathrm{H}_{\alpha}$ emission/absorption is not - and must not be present (Hen 36 and 45); (iii) extrinsic stars with weak residual emission likely due to a slight mismatch between the template spectrum (Hen 6) and the target spectra (Hen 138 and 129; see Fig. 7 for more profiles of extrinsic stars); (iv) extrinsic stars with weak symbiotic-like profiles (Hen 134 and 137); (v) symbiotic S stars (Hen 18 and 121). The $\mathrm{H}_{\alpha}$ rest wavelength is indicated by a dashed line. 


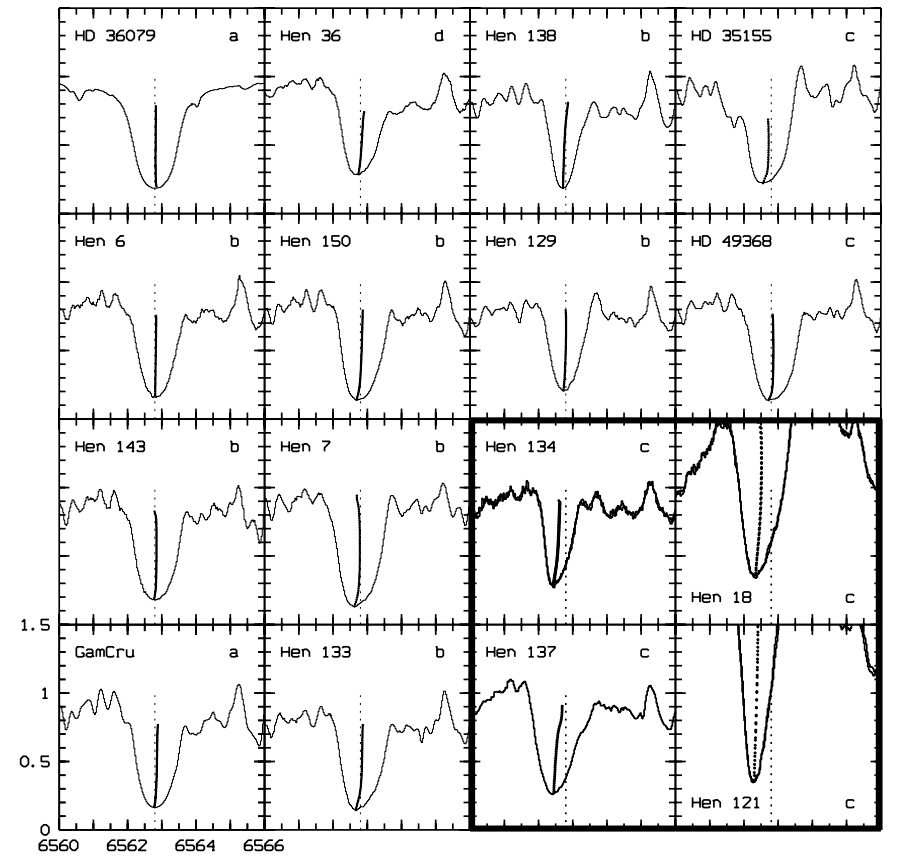

Fig. 6. Representative examples of $\mathrm{H}_{\alpha}$ profiles and their associated bisectors. All panels are drawn on the same scale, as indicated on the lower left panel. All spectra have been corrected from the Doppler shift, and the $\mathrm{H}_{\alpha}$ laboratory wavelength is depicted by the vertical dashed line. The label in the upper right corner of each panel classifies the star according to the following categories: a: radial-velocity standard star; b: extrinsic S star; c: extrinsic symbiotic S star (see also Table 2); d: intrinsic S star. The leftmost column corresponds to stars with an almost vertical bisector; the second to leftmost column displays stars with a bisector slightly slanted under the influence of the TiO absorptions falling in the red wing of the $\mathrm{H}_{\alpha}$ line; stars with a definite symbiotic signature in their $\mathrm{H}_{\alpha}$ profile, as discovered in the present study, lie within the bold frame.

of Fig. 6). TiO lines mutilating the red wing of the $\mathrm{H}_{\alpha}$ line - as confirmed by synthetic spectra - are responsible for the slanted appearance of the bisectors in the second to leftmost column in Fig. 6. This column includes intrinsic S stars and cool extrinsic $\mathrm{S}$ stars where $\mathrm{TiO}$ bands are strong. In all these cases, the bisector in the core of the line is nevertheless located very close to the $\mathrm{H}_{\alpha}$ wavelength, as expected. On the contrary, the bisectors of Hen 18 and 121 are strongly blueshifted in the line core. A similar trend is also clearly apparent in the case of Hen 134 and 137, which we therefore tag as weak symbiotic stars. All S stars for which our $\mathrm{H}_{\alpha}$ profiles are indicative of symbiotic activity have been collected in the four bold frames of Fig. 6. The analysis of the normalized $\mathrm{H}_{\alpha}$ profiles (Fig. 5) and of the line bisector (Fig. 6) thus yields the same set of symbiotic $S$ stars among the Henize sample.

\subsection{Correlation of symbiotic activity with orbital period}

The present data offer a unique opportunity to correlate the symbiotic activity (diagnosed by the presence of $\mathrm{H}_{\alpha}$ emission) with the orbital period (Fig. 7). Neither the binaries with the shortest periods nor those with the longest do exhibit any $\mathrm{H}_{\alpha}$ emission. Symbiotic activity seems instead restricted to a rather
Table 2. Presence or absence of symbiotic activity in $S$ stars as a function of orbital period, according to various diagnostics: X-rays ("X"), UV continuum (in column "UV cont.", " $y$ " means that excess UV flux is present, but does not match a clean WD spectrum), C IV $\lambda 155 \mathrm{~nm}$ and $\mathrm{Mg}$ II $\lambda 280 \mathrm{~nm}$ lines, $\mathrm{H}_{\alpha}$ emission, and $\mathrm{He} \mathrm{I} \lambda 1083 \mathrm{~nm}$. The stars have been ordered according to increasing orbital period.

\begin{tabular}{lccccccc}
\hline \hline Name & $\begin{array}{r}P \\
\text { (d) }\end{array}$ & $\begin{array}{c}\text { X } \\
\text { cont. }\end{array}$ & $\begin{array}{c}\text { UV } \\
\text { cIV }\end{array}$ & $\begin{array}{c}\text { MgII } \\
\lambda 280\end{array}$ & $\begin{array}{c}\mathrm{H}_{\alpha} \\
\text { em. }\end{array}$ & $\begin{array}{c}\text { He I } \\
\lambda 1083\end{array}$ \\
\hline Hen 108 & 197 & $?$ & $?$ & $?$ & $?$ & no & $?$ \\
Hen 147 & 335 & $?$ & $?$ & $?$ & $?$ & no & $?$ \\
HR 1105 & 596 & $?$ & y & y & y & $?$ & var \\
Hen 137 & 636 & $?$ & $?$ & $?$ & $?$ & moderate & $?$ \\
HD 35155 & 642 & var? & y & y & y & moderate? & var \\
Hen 121 & 764 & $?$ & y & $?$ & $?$ & strong & $?$ \\
HD 191226 & 1210 & $?$ & WD & no & y & $?$ & wk em. \\
Hen 119 & 1300 & $?$ & $?$ & $?$ & $?$ & no & $?$ \\
HD 49368 & 3000 & $?$ & y & y & y & no & absorption \\
HR 363 & 4590 & var & y & no & $?$ & $?$ & wk em. \\
\hline Hen 18 & $?$ & $?$ & y & $?$ & $?$ & strong & $?$ \\
Hen 134 & $?$ & $?$ & $?$ & $?$ & $?$ & moderate & $?$ \\
ER Del & $?$ & $?$ & $?$ & $?$ & $?$ & strong & $?$ \\
\hline Ref & 1 & 2 & 3 & 3 & 4 & 5 & 6 \\
\hline
\end{tabular}

References: (1) Jorissen et al. (1998, A\&A, 332, 877); Griffin (1984, The Observatory, 104, 224); Carquillat et al. (1998, A\&AS, 131, 49); Paper II (2) Jorissen et al. (1996, A\&A, 306, 467); (3) Johnson et al. (1993, ApJ, 402, 667 and references therein); Ake (1996, priv. comm.); (4) Ake et al. (1994. In: A.W. Shafter (ed.) Interacting Binary Stars (ASP Conf. Ser. 56)); Ake (1996, priv. comm.); (5) Johnson \& Ake (1989. In: H.R. Johnson, B. Zuckerman (eds.) Evolution of Peculiar Red Giants (IAU Coll. 106) Cambridge Univ. Press, p. 371); Ake et al. (1991, ApJ, 383, 842; this work); (6) Brown et al. (1990, AJ, 99, 1930); Shcherbakhov \& Tuominen (1992, A\&A, 255, 215).

narrow period range around $800 \mathrm{~d}( \pm 200 \mathrm{~d}$; see Table 2$)$. This conclusion is consistent with the distribution of orbital periods known so far among Henize S stars. There are 8 orbital periods available (Table 1 of Paper II), among which only 3 (Hen 121, $137,183)$ fall in the symbiotic period range. Two of them are indeed symbiotics. The third one (Hen 183) does not seem to show any sign of symbiotic activity (normal $U B V$ color indices but no data available on $\mathrm{H}_{\alpha}$; Paper II); its case is further discussed below. The other 5 systems have orbital periods either shorter or larger than the symbiotic period range. Since binaries with short periods are the easiest to discover, it is likely that all short-period systems existing among the Henize extrinsic $S$ stars have been found. Thus, the small number of symbiotics among the Henize sample just reflects their orbital-period distribution.

The reason why symbiotic $S$ stars seem restricted to the period range 600-1000 d should now be understood, and in particular why systems with shorter orbital periods are not symbiotics. This question may actually be addressed by identifying the parameters required to trigger symbiotic activity (see also the discussion in Jorissen 2003). The key factor in this respect is the high luminosity of the compact companion, that should exceed $10 L_{\odot}$ (e.g., Yungelson et al. 1995), in order to provide 


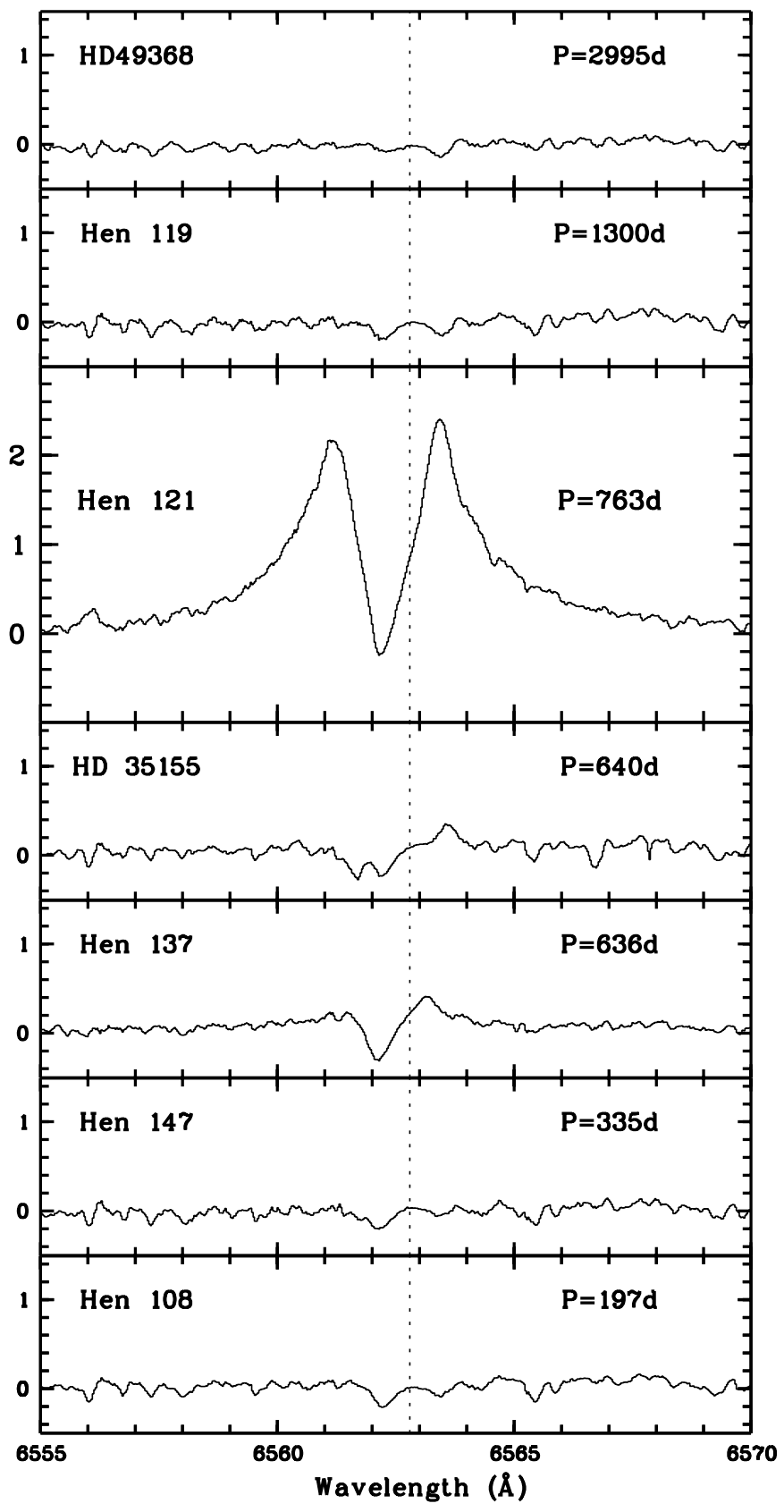

Fig. 7. Same as Fig. 5 for binary S stars, ordered from top to bottom according to decreasing orbital periods (as indicated in the upper right corner). It is clearly apparent that $\mathrm{S}$ stars with $\mathrm{H}_{\alpha}$ emission are found in the period range $600-1000 \mathrm{~d}$.

the high-energy photons necessary to excite atoms in the redgiant wind. This requirement in turn sets a constraint on the accretion rate by the compact star, which should be large enough to deliver such a luminosity, either through nuclear burning at the white dwarf surface or directly through accretion luminosity. Detailed hydrodynamical simulations of mass transfer in symbiotic binaries (Theuns et al. 1996; Mastrodemos \& Morris 1998) have shown that the Bondi-Hoyle accretion regime generally predicts accretion rates that are an order of magnitude too large. It provides however a convenient analytical

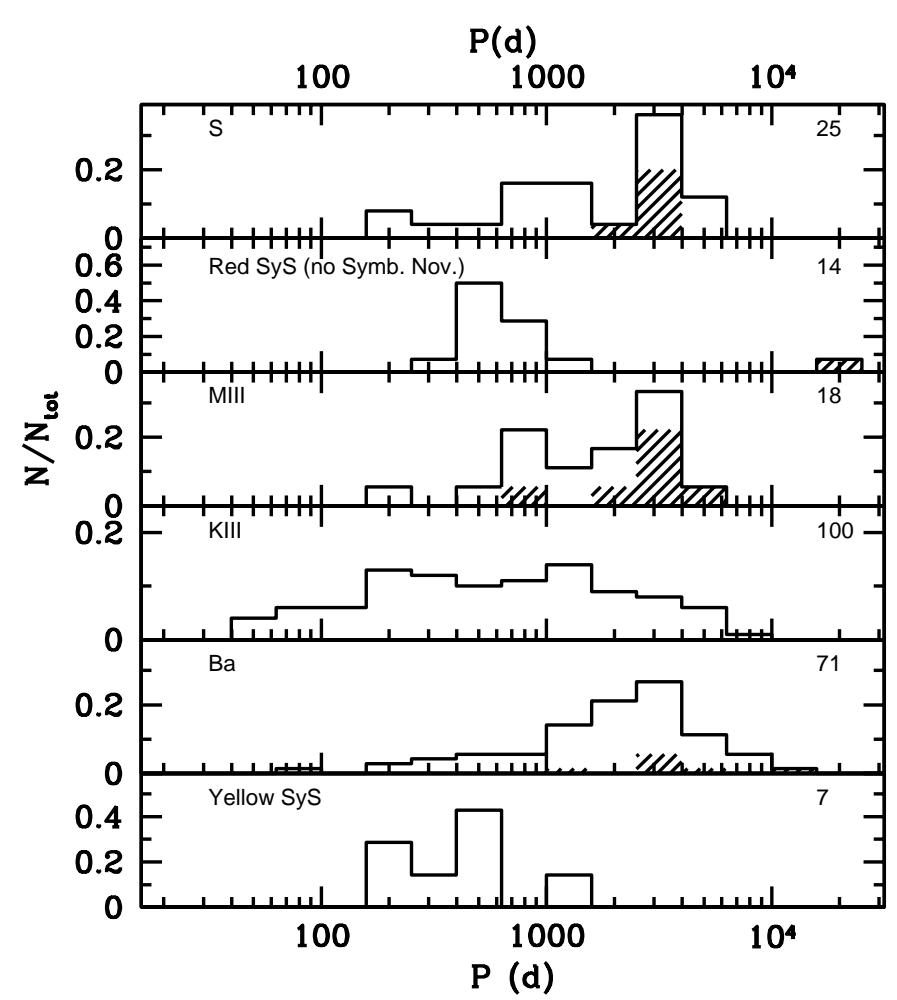

Fig. 8. Comparison of the orbital period distributions for S stars (Jorissen et al. 1998), red symbiotics (excluding symbiotic novae; Mürset \& Schmid 1999), K giants (Mermilliod 1996) and M giants (Jorissen et al., in preparation). The lower two panels present the orbital-period distribution for barium stars (Jorissen et al. 1998) and yellow symbiotics (Mürset \& Schmid 1999). The shaded area marks stars with only a lower limit on their orbital period. The numbers in the upper right corner of each panel correspond to the sample size.

formulation of the mass accretion rate that may be used to identify key parameters:

$$
\begin{aligned}
\dot{M}_{\mathrm{acc}, B-H}= & -\dot{M}_{\text {wind }} \frac{\eta}{A^{2}}\left(\frac{G M_{2}}{v_{\text {wind }}^{2}}\right)^{2} \\
& \times \frac{1}{\left[1+\left(\frac{v_{\text {orb }}}{v_{\text {wind }}}\right)^{2}+\left(\frac{c}{v_{\text {wind }}}\right)^{2}\right]^{3 / 2}}
\end{aligned}
$$

where $M_{2}$ is the mass of the accreting star, $\eta \sim 1$ if the BondiHoyle accretion regime applies (i.e., $v_{\text {wind }} / v_{\text {orb }} \gg 1$ ), $\eta \sim 0.1$ otherwise (if $v_{\text {wind }} / v_{\text {orb }} \lesssim 1$ ).

Equation (1) thus shows that three parameters are of prime importance in fixing the accretion rate: the wind velocity of the giant star $v_{\text {wind }}$, which according to van Loon (2000) is proportional to $L_{1}^{1 / 4} \sqrt{Z}$ ( $Z$ is the metallicity and $L_{1}$ the luminosity of the giant), the orbital period $P$ which is related to both the orbital separation $A$ and the orbital velocity $v_{\text {orb }}$, and the wind mass loss rate $\dot{M}_{\text {wind }}$.

The supposedly low mass-loss rates characterizing S stars in short-period systems probably account for their absence of symbiotic activity. Indeed, only compact, i.e, warm and low-luminosity giants may be hosted by the shortest-period (200-500 d) systems. More evolved giants are too big to hold within their Roche lobe in short-period systems. 


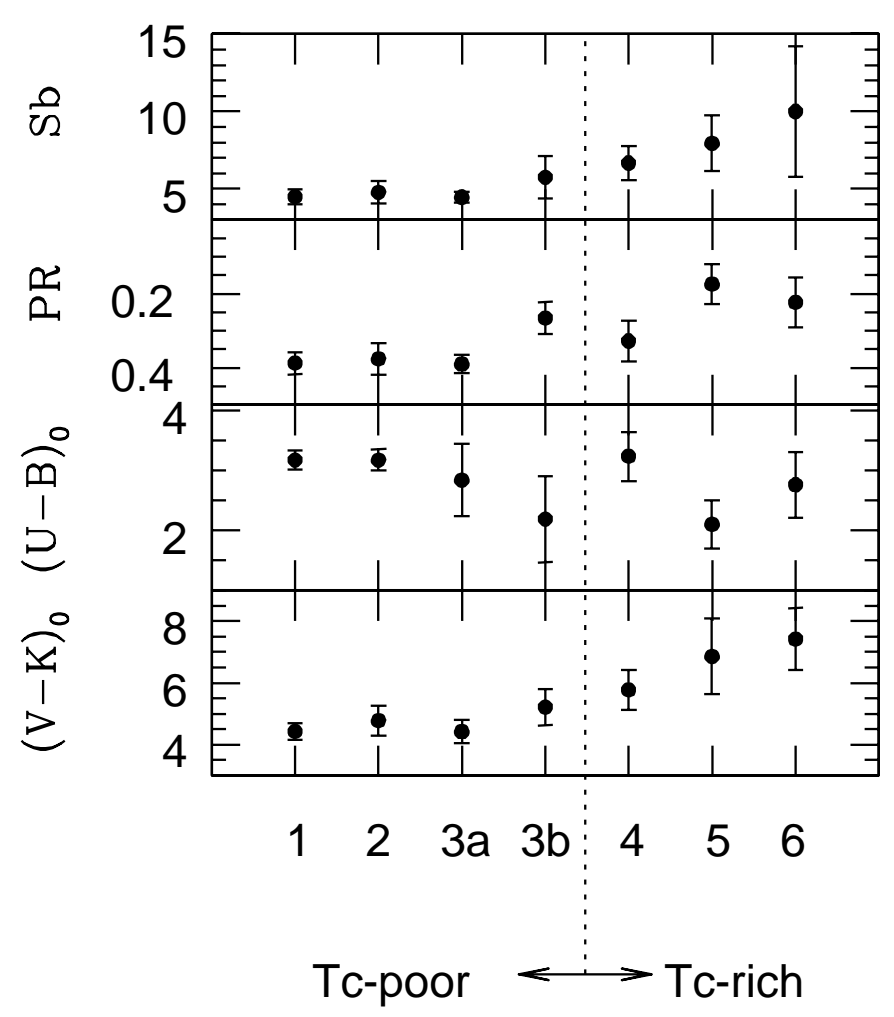

Fig. 9. Comparison of luminosity and temperature indicators for binary S stars (groups 1, 2 and 3) and intrinsic S stars (groups 4, 5 and 6; see Paper III for a detailed description of these groups). Symbiotic S stars (Hen 18, 121, 134, 137 and HD 35155) form group 3b, whereas binary $\mathrm{S}$ stars with no $\mathrm{H}_{\alpha}$ emission (Hen 108, 119, 147 and HD 49368) constitute group $3 \mathrm{a}$. The color index $V-K$ is a good temperature indicator, whereas the CORAVEL parameters $S b$ (average spectral line width, in $\mathrm{km} \mathrm{s}^{-1}$, corrected for the instrumental width) and $P R$ (average line depth) are good luminosity indicators. The symbiotic $\mathrm{S}$ stars have temperature and luminosity indicators that are the most extreme among binary $\mathrm{S}$ stars, and are close to the values characterizing intrinsic, more evolved, S stars. On the contrary, binary S stars with no $\mathrm{H}_{\alpha}$ emission (group 3a) are clearly much less evolved than the symbiotic $\mathrm{S}$ stars. For comparison the deredenned $U-B$ color index is also shown.

This statement is confirmed by the empirical correlation between spectral types and orbital periods observed among symbiotic systems by Mürset \& Schmid (1999), late spectral types being restricted to systems with the longest periods. It is also apparent from the comparison of the period distributions of $\mathrm{K}$ and $\mathrm{M}$ giants (Fig. 8): no system with an orbital period shorter than about $400 \mathrm{~d}$ is observed among $\mathrm{M}$ giants, whereas the lower period cutoff for $\mathrm{K}$ giants is only about $40 \mathrm{~d}$.

Figure 8 moreover reveals that the period range $600-1000 \mathrm{~d}$ where most symbiotic $\mathrm{S}$ stars are found is also where most red symbiotic stars are located (when excluding symbiotic novae and symbiotic Miras). Those red symbiotic stars are in turn found in the short-period tail of the distribution of M giants. Similarly, yellow symbiotic stars occupy the short-period tail of the distribution of Ba stars (Fig. 8). Symbiotic stars are thus found among binaries having the shortest allowed periods given the evolutionary stage of the cool component. Binary $\mathrm{S}$ stars with the longest orbital periods do not exhibit $\mathrm{H}_{\alpha}$ emission (Fig. 7 and Table 2) since, for a given mass loss rate, the accretion rate is smaller in wider systems (Eq. (1)). Table 2 reveals, however, that very long-period systems like HR 363 and HD 49368 nevertheless exhibit other signatures of symbiotic activity (for instance, in the form of X-rays or UV continuum), despite the absence of $\mathrm{H}_{\alpha}$ emission. This is not surprising since $\mathrm{H}_{\alpha}$ emission is not as sensitive a diagnostic of symbiotic activity as are the UV or X-ray emissions, as confirmed for instance by the absence of $\mathrm{H}_{\alpha}$ emission at some epochs for the classical - albeit rather weak - symbiotic star EG And.

Figure 9 compares the properties of intrinsic $\mathrm{S}$ stars, symbiotic $\mathrm{S}$ stars and non-symbiotic extrinsic $\mathrm{S}$ stars, and provides an indirect confirmation of the above qualitative conclusion that symbiotic $\mathrm{S}$ stars ought to lose more mass than nonsymbiotic, binary $\mathrm{S}$ stars, and thus presumably be somewhat more evolved. The $V-K$ index is a good temperature indicator, and Fig. 9 indicates that the symbiotic $\mathrm{S}$ stars (group $3 \mathrm{~b}$ in Fig. 9) are among the coolest extrinsic S stars. Observable diagnostics of luminosity include the CORAVEL $S b$ and $P R$ parameters, which measure the average line width and depth, respectively (see Paper II for a more detailed description of these indicators). Figure 9 shows that the symbiotic $\mathrm{S}$ stars have values of $S b$ and $1-P R$ that are the largest among binary $\mathrm{S}$ stars, even close to those characterizing the more evolved intrinsic $\mathrm{S}$ stars. Thus, both the temperature and luminosity indicators suggest that the symbiotic $\mathrm{S}$ stars are the most evolved among the binary S stars, and thus may indeed be expected to suffer from the largest mass loss rates. The above analysis also justifies why Hen 183 does not show any sign of symbiotic activity, despite an orbital period of about $890 \mathrm{~d}$. With $S b=4.7 \mathrm{~km} \mathrm{~s}^{-1}$, $P R=0.37$ and $(V-K)_{0}=4.77$, Hen 183 appears somewhat less evolved than symbiotic S stars (Fig. 9).

\section{Conclusions}

Two strong (Hen 18 and Hen 121) and two weak (Hen 134 and Hen 137) symbiotic stars were found among the binary S stars from the Henize sample, as revealed by their strong or moderate $\mathrm{H}_{\alpha}$ emission. Their $\mathrm{H}_{\alpha}$ profiles are typical of symbiotic stars, more precisely of class S-3 as defined by Van Winckel et al. (1993). Hen 18 and Hen 121 are also characterized by very blue $B-V$ and $U-B$ indices. The eclipse of the Balmer ultraviolet continuum observed for Hen 121 clearly indicates that the emission region is tied to the companion. The symbiotic $\mathrm{S}$ stars, when diagnosed by their $\mathrm{H}_{\alpha}$ emission, are restricted to the period range 600-1000 d, also typical of red symbiotic stars (excluding symbiotic novae and symbiotic Miras), and which represents the short-period tail of the distribution of binary $M$ giants. Moreover, there is compelling evidence that symbiotic $\mathrm{S}$ stars are somewhat more evolved than non-symbiotic binary $\mathrm{S}$ stars. These properties may be easily understood since the cool components in symbiotic systems need to lose mass at a rate large enough to power the high luminosity $\left(L>10 L_{\odot}\right)$ of the compact companion and thereby to trigger the observed symbiotic activity.

Acknowledgements. We thank G. Burki and M. Burnet for securing the photometric observations, M. Kürster for help with the 
computation of line bisectors, and H.M. Schmid for enlightening discussions about the physics of symbiotic stars.

\section{References}

Arkhipova, V. P., \& Noskova, R. I. 1985, Sov. Astron. Lett., 11, 297 Grenon, M. 1978, Ph.D. Thesis, Observatoire de Genève Jorissen, A. 2003, in Symbiotic stars probing stellar evolution, ed. R. Corradi, \& J. Mikołajewska (Astron. Soc. Pacific Conf. Ser., San Francisco), in press

Jorissen, A., Mayor, M., Manfroid, J., \& Sterken, C. 1992, Information Bulletin on Variable Stars, 3730, 1

Jorissen, A., Van Eck, S., Mayor, M., \& Udry, S. 1998, A\&A, 332, 877

Kenyon, S. J. 1986, Symbiotic stars (Cambridge University Press, Cambridge)

Lee, H. 2000, ApJ, 541, L25

Mastrodemos, N., \& Morris, M. 1998, ApJ, 497, 303

Mermilliod, J.-C. 1996, in The Origins, Evolution, and Destinies of Binary Stars in Clusters, ed. E. Milone, \& J.-C. Mermilliod, ASP Conf. Ser., 90, 95

Mürset, U., Dumm, T., Isenegger, S., et al. 2000, A\&A, 353, 952

Mürset, U., \& Schmid, H. M. 1999, A\&AS, 137, 473

Pereira, C. B., Cunha, K., \& Smith, V. V. 2003, in Symbiotic stars probing stellar evolution, ed. R. Corradi, \& J. Mikołajewska (Astron. Soc. Pacific Conf. Ser., San Francisco), in press

Pereira, C. B., \& Porto de Mello, G. F. 1997, AJ, 114, 2128
Pereira, C. B., Smith, V. V., \& Cunha, K. 1998, AJ, 116, 1977

Plez, B., Van Eck, S., Jorissen, A., et al. 2003, in Modelling of Stellar Atmospheres, ed. N. E. Piskunov, W. W. Weiss, \& D. F. Gray, IAU Symp. 210 (ASP, San Francisco), in press

Robinson, K., Bode, M. F., Skopal, A., Ivison, R. J., \& Meaburn, J. 1994, MNRAS, 269, 1

Schmid, H. M. 1994, A\&A, 284, 156

Schwank, M., Schmutz, W., \& Nussbaumer, H. 1997, A\&A, 319, 166

Smith, V. V., Cunha, K., Jorissen, A., \& Boffin, H. M. J. 1996, A\&A, 315,179

Smith, V. V., Cunha, K., Jorissen, A., \& Boffin, H. M. J. 1997, A\&A, 324, 97

Smith, V. V., Pereira, C. B., \& Cunha, K. 2001, ApJ, 556, L55

Stephenson, C. B. 1984, A General Catalogue of Galactic S stars, Second Edition, Publications of the Warner and Swasey Observatory, 3, 1, Columbus

Theuns, T., Boffin, H. M. J., \& Jorissen, A. 1996, MNRAS, 280, 1264

Van Eck, S., \& Jorissen, A. 2000a, A\&AS, 145, 51 (Paper II)

Van Eck, S., \& Jorissen, A. 2000b, A\&A, 360, 196 (Paper III)

Van Eck, S., Jorissen, A., Udry, S., Mayor, M., \& Pernier, B. 1998, A\&A, 329, 971

van Loon, J. T. 2000, A\&A, 354, 125

Van Winckel, H., Duerbeck, H. W., \& Schwarz, H. E. 1993, A\&AS, 102,401

Woodsworth, A. W. 1995, ApJ, 444, 396

Yungelson, L., Livio, M., Tutukov, A., \& Kenyon, S. J. 1995, ApJ, 447,656 